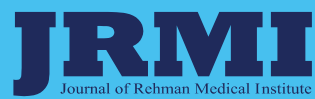

Volume 6, No. 3

July - September 2020

www.jrmi.pk

\title{
Trends in antibiotic susceptibility of enteric fever isolates among children attending a tertiary care hospital of Peshawar, KP
}

Samreen Ahmad, Shahzada Bakhtyar Zahid, Abid Salahuddin, Adnan Khan, Muhammad Hassaam Khan, Husnul Maab Ali

\section{Submitted}

July 18,2020

Accepted

August 25, 2020

\section{Author Information}

From: Department of Pediatrics, Rehman Medical Institute, Peshawar, Khyber Pakhtunkhwa, Pakistan

Dr Samreen Ahmad Consultant Pediatrician (Corresponding Author) Email:

samreen.ahmad@rmi.edu.pk

Prof Dr. Shahzada Bakhtyar Zahid

HoD \& Consultant

Pediatrician

Dr. Abid Salahuddin

Consultant Pediatrician

Dr Adnan Khan

Trainee Medical Officer

Dr Muhammad Hassam Khan House Officer

Dr Husnul Maab Ali

House Officer

Citation: Ahmad S, Zahid SB, Salahuddin A, Khan A, Khan MH, Ali HM. Trends in antibiotic susceptibility of enteric fever isolates among children attending a tertiary care hospital of Peshawar, KP. J Rehman Med Inst. 2020 Jul-Sep;6(3):20-3.

\begin{abstract}
Introduction: The rising trend of multi-drug resistance in bacteria has also been documented in the organism Salmonella enterica causing Typhoid or Enteric Fevers, and cases of multidrug and extended drug resistance are on the rise. Determining the trends of antibiotic susceptibility provides an important therapeutic aid to the practicing clinician.
\end{abstract}

Objective: To evaluate the ten-year trends in antibiotic susceptibilities of Salmonella typhi and Salmonella paratyphi isolated from blood cultures in the paediatric patients of a tertiary care hospital of Peshawar, Khyber Pakhtunkhwa, Pakistan.

Materials \& Methods: A descriptive study was conducted in the department of Paediatrics, Rehman Medical Institute, Peshawar from June 2019 to May 2020 based on ten years retrospective data of children with positive blood culture for Salmonella spp. Data were analyzed using IBM SPSS Statistics for Windows, Version 20.0 (IBM Corp., Armonk, NY). The level of significance was set at $\mathrm{p} \leq 0.05$.

Results: A total of 168 cases Salmonella enterica spp. were isolated over the 10-year study period, of which 97(64.88\%) isolates were identified as Salmonella typhi and $71(42.26 \%)$ as Salmonella paratyphi A; 94(55.95\%) patients were male and $74(44.04 \%)$ were female, with mean age of $4.76 \pm 2.65$ years (range 2 to 12 years); majority, 69(41.07\%) of patients were of ages 5-8 years. $S$. typhi strains showed the highest sensitivity to Imipenem and Meropenem; the lowest sensitivity seen was to Ampicillin. Bacterial sensitivity to Ceftriaxone was $79.76 \%$ and to Ciprofloxacin $58.97 \%$. In the $S$. typh group, there were six (2.6\%) cases of MDR typhoid and four $(2.38 \%)$ cases of XDR typhoid which was only sensitive to Imipenem.

Conclusion: Increased resistance to Ampicillin, Ciprofloxacin and Ceftriaxone was found, however complete sensitivity was found to Imipenem along with Meropenem.

The authors declared no conflict of interest. All author contributed substantially to the planning of research, data collection, data analysis, and write-up of the article, and agreed to be accountable for all aspects of the work.

\section{INTRODUCTION}

Enteric Fever (commonly called Typhoid fever), a disease endemic to tropical and subtropical regions, is caused by the bacterium Salmonella enterica serovar typhi. It is spread by feco-oral route and is common globally, especially in underdeveloped countries due to contamination of food and liquids with salmonella species. Typhoid fever is related to poor hygiene and sanitation, and is among the common febrile illnesses, involving multiple body systems with serious complications. Symptoms might range from mild to moderate or severe, and so are its complications, which might prove fatal if left untreated. This disease is mostly thought of as a disease of developing countries because of poor sanitary conditions. ${ }^{1}$ Typhoid fever has been mostly eradicated from developed countries due to improved sanitary and hygienic conditions. ${ }^{2}$ With an estimated annual incidence of 540 per 100,000 or about 17 million cases worldwide, ${ }^{3}$ it is a major public health problem, and accounts for a sizable number of cases of global morbidity and mortality. ${ }^{4}$

The causative organism, Salmonella typhi, has rapidly gained Multidrug Resistance (MDR) and now Extended Drug Resistance (XDR) to antibiotics like Ampicillin, Ceftriaxone, and Cotrimoxazole, and also to previously efficacious drugs like Ciprofloxacin, ${ }^{5}$ thereby further complicating the treatment and management of enteric fever, and posing the greatest challenges to paediatric consultants in managing paediatric Salmonella typhi infections. In 2017, Global Health Data Exchange reported approximately 116,800 deaths due to typhoid fever. Of these there were about 79,000 deaths in South Asia, with 6,700 reported from Pakistan. ${ }^{6}$

The incidence of MDR typhoid is reported to be $17 \%$ to $23 \%$ in South Asia which led to shift from first-line antityphoid drugs to first- and thirdgeneration Cephalosporins and Fluoroquinolones (FQ) (second-line agents for typhoid), and azithromycin. ${ }^{7}$ Concurrently, newer studies have started to report increasing antimicrobial resistance to one or more second-line antityphoid agents. ${ }^{8}$ 
Contributory factors to emergence of antibiotic resistance include monotherapy, incomplete directions for antibiotic use, cessation of antibiotic therapy by patients, and prescriptions given without biochemical testing. Finally, in 2016, the first case of XDR typhoid was reported from Hyderabad, a small city in the South of Pakistan. XDR typhoid is defined as the isolate resistant to all first-line antityphoid agents, Ceftriaxone (CFX), and FQ. ${ }^{9}$ Since then, the World Health Organization has reported more than 5,200 cases of XDR typhoid from Pakistan, all of which were from Southern Pakistan. The present study was done to evaluate the current trends in antibiotic susceptibilities of S. typhi and S. paratyphi isolated from blood cultures in the paediatric population of Peshawar, Pakistan.

\section{MATERIALS \& METHODS}

An observational study was conducted at Department of Paediatrics, Rehman Medical Institute, Peshawar, retrospective data of all patients with positive blood culture for salmonella in the last 10 year from RMI database through consecutive sampling. Inclusion criteria were children age less than 18 years, children with positive blood culture for salmonella, and both genders. Exclusion criteria were incomplete or ambiguous relevant records.

\section{Sample collection procedure}

For each child, $5 \mathrm{~mL}$ of venous blood was taken and collected in special blood culture bottles containing $30 \mathrm{~mL}$ brain heart infusion (BHI) broth and was incubated for five to seven days at $35 \pm 2{ }^{\circ} \mathrm{C}$. Blood samples that were positive for $\mathrm{S}$. typhi or $\mathrm{S}$. paratyphi were subcultured on McConkey agar for another two days at $35 \pm 2^{\circ} \mathrm{C}$ as per standard methods. API-20E (Biomerieux, France), the analytical profile index system specific for differentiating between members of Gram-negative Enterobacteriaceae, was utilized for biochemical testing. Serotyping was done by group and type-specific antisera (BioRad). Kirby-Bauer disc diffusion technique on Mueller Hinton agar was utilized to determine the patterns of antimicrobial sensitivity. The agar plated will be incubated aerobically, at 35 $\pm 2^{\circ} \mathrm{C}$, for 24 hours.

\section{Data analysis}

Data were analyzed using IBM SPSS Statistics for Windows, Version 20.0 (IBM Corp., Armonk, NY). Continuous variables were reported as mean and standard deviation and categorical variables as number (percent). The level of significance was set at $\mathrm{P} \leq 0.05$.

\section{RESULTS}

Of the total 168 Salmonella enterica spp. isolated over the 10year study period, 97(64.88\%) isolates were identified as Salmonella typhi and 71(42.26\%) as Salmonella paratyphi A; Among patients, 94(55.95\%) were male and 74(44.04\%) were female, with mean age of $4.76 \pm 2.65$ years, ranging from 2 to 12 years. Majority $69(41.07 \%$ ) of the patients were in age group 5-8 years. The frequency and percentages of $S$. typhi and $S$. paratyphi strain according to the age and gender of the children are shown in Table 1.

Table 1: Demographic characteristics of the study population

\begin{tabular}{|c|c|c|c|}
\hline Variables & $\begin{array}{c}\text { Salmonella } \\
\text { typhi }(\mathbf{n = 9 7})\end{array}$ & $\begin{array}{c}\text { Salmonella paratyphi } \\
(\mathbf{n}=\mathbf{7 1})\end{array}$ & $\begin{array}{c}\text { Total } \\
(\mathbf{n}=\mathbf{1 6 8})\end{array}$ \\
\hline Gender & & & \\
\hline Male & $56(57.73 \%)$ & $38(53.52 \%)$ & $94(55.95 \%)$ \\
\hline Female & $41(42.26 \%)$ & $33(46.47 \%)$ & $74(44.04 \%)$ \\
\hline Age group (years) & & & \\
\hline $2-5$ & $14(14.43 \%)$ & $9(12.67 \%)$ & $23(32.39 \%)$ \\
\hline $5-8$ & $41(42.26 \%)$ & $28(39.43 \%)$ & $69(97.18 \%)$ \\
\hline $8-11$ & $27(27.83 \%)$ & $8(11.26 \%)$ & $35(49.29 \%)$ \\
\hline $11-14$ & $29(29.89 \%)$ & $12(16.90 \%)$ & $41(57.74 \%)$ \\
\hline
\end{tabular}

The most frequent presenting concern among the study sample was non focal pyrexia $(87,51.8 \%)$ and one child complicated into intestinal perforation which was surgically managed. A previous trial of antibiotics was taken by 97(58.9\%) children.
The most common antibiotics taken were Cefixime by 86 $(51.2 \%)$ children. The antimicrobial sensitivity patterns are shown in Table 2.

Table 2: Showing antibiotic sensitivities of Salmonella typhi and paratyphi.

\begin{tabular}{|l|c|c|}
\hline \multicolumn{1}{|c|}{ Antibiotic } & $\begin{array}{c}\text { Salmonella typhi }(\mathbf{n = 9 7}) \\
\mathbf{f}(\%)\end{array}$ & $\begin{array}{c}\text { Salmonella paratyphi }(\mathbf{n = 7 1}) \\
\mathbf{f}(\boldsymbol{\%})\end{array}$ \\
\hline Ampicillin & $48(49.48)$ & $23(32.39)$ \\
\hline Ciprofloxacin & $57(58.76)$ & $36(50.70)$ \\
\hline Chloramphenicol & $53(54.63)$ & $32(45.07)$ \\
\hline Ceftriaxone & $83(85.56)$ & $51(71.83)$ \\
\hline Azithromycin & $93(95.87)$ & $67(94.36)$ \\
\hline Imipenem & $97(100)$ & $71(100)$ \\
\hline Meropenem & $97(100)$ & $71(100)$ \\
\hline
\end{tabular}


S. typhi strains showed the highest sensitivity to Imipenem and Meropenem; the lowest sensitivity seen was to Ampicillin. Bacterial sensitivity to Ceftriaxone was $79.76 \%$ which is alarming since these are commonly used empirical agents against salmonella. Only half of the S. typhi isolates showed sensitivity to ciprofloxacin $(58.97 \%)$ which is also a rising concern. In the S. typhi group, there were six (2.6\%) cases of MDR typhoid and four (2.38\%) cases of XDR typhoid which was only sensitive to Imipenem.

\section{DISCUSSION}

In Pakistan enteric fever is a growing concern, due to emergence of multidrug resistant salmonella in the last few years. The worldwide emergence of MDR strain of salmonella in last decade has led to withdrawal of certain antibiotics, like replacement of Chloramphenicol with Fluroquinolones and Ceftriaxone. However, recently the efficacy of Ciprofloxacin as well as Ceftriaxone in Pakistan for enteric treatment has been seriously jeopardised. ${ }^{10}$

In our study, the prevalence of S. typhi was $64.8 \%$ and for paratyphi it was $42.26 \%$. Majority $69(41.07 \%$ ) of the patients were in age group 5-8 years. Globally in all microbes the antimicrobial resistance is increasing at an alarming rate. Research data shows that MDR typhoid prevalence rose from $12.14 \%$ in 1987 to $75.41 \%$ in 1995 in Pakistan. ${ }^{11}$ Another study reported MDR prevalence in Asian countries at 23\%. ${ }^{12}$ Hassan et al. conducted a study in which they showed MDR rates of $34.2 \%$ to $48.5 \%$ for Typhi from 2001 to $2006 .{ }^{13}$ The Quinolone resistance in S. typhi and paratyphi A rose dramatically (1.6 to $64.1 \%, \mathrm{p}<0.001$; and 0 to $47 \%, \mathrm{p}<0.001$, respectively). Current data on MDR shows that it is prevalent up to $66 \%, 28.6 \%$ and $64.28 \%$ in Pakistan, India and Bangladesh, respectively.
Rahman BA et al, ${ }^{14}$ showed that among Middle East and Central Asian countries the MDR prevalence is highest in Iraq (83\%) and Pakistan (52\%).

In our study resistance was found to Ampicillin and Ciprofloxacin and to third generation Cephalosporin. This was supported by other studies in literature from Pakistan and other neighbouring countries. ${ }^{15,16}$ A recent study from developing countries showed resistance of against Chloramphenicol and Ciprofloxacin was observed. ${ }^{17}$ In the late 2000, since the 1 st line antibiotics for Salmonella Typhi showed resistance, the Fluoroquinolones and 3rd generation of Cephalosporin had been used in many endemic places and countries including Pakistan. However, shortly after the frequent use of Fluoroquinolones, Salmonella typhi resistance to this group of antibiotic has widely reported. Variation in the susceptibility patterns of Salmonella typhi against antibiotics is worth noting.

Our study showed that sensitivity to Imipenem and Meropenem to typhoid isolates was highest. Bacterial sensitivity to Ceftriaxone is $79.76 \%$. One half of Salmonella typhi showed sensitivity to Ciprofloxacin. Imipenem and Meropenem showed 97\% sensitive in our study. Due to the emerging resistance to a wide range of antimicrobials in Asian countries, the cost of typhoid infection has increased much over the years. This cost can be reduced by effective immunisation as recommended by the WHO and improvement in sanitation in developing countries.

\section{CONCLUSION}

Increased resistance of Salmonella spp. was found to Ampicillin, Ciprofloxacin and Ceftriaxone, however there was complete sensitivity to Imipenem along with Meropenem.

\section{REFERENCES}

1. World Health Organization (WHO), Prepared for World Water Day 2001. Reviewed by Staff and Experts from the Cluster on Communicable Diseases (CDS) and the Water, Sanitation and Health Unit (WSH), World Health Organization (WHO), 2008, http://www.who.int/vaccine_research/dis eases/diarrhoeal/en/index7.html.

2. E. S. Anderson and H. P. Smith, "Precise estimation of the number of chronic carriers of Salmonella typhi in Santiago, Chile, an endemic area," British Medical Journal, vol. 3, pp. 329-331, 2002

3. Neopane, S. B. Singh, R. Bhatta, B. Dhital, and D. B. Karki, "Changing spectrum of antibiotic sensitivity in enteric fever," Kathmandu University Medical Journal, vol. 6, no. 1, pp. 12-15, 2008

4. C. Ibekwe, I. O. Okonko, A. U. Onunkwo, E. Donbraye, E. T. Babalola, and B. A. Onoja, "Baseline Salmonella agglutinin titres in apparently healthy freshmen in Awka, South Eastern,
Nigeria," Scientific Research and Essays, vol. 3, no. 9, pp. 225-230, 2008

5. T. Butt, R. N. Ahmad, A. Mahmood, and S. Zaidi, "Ciprofloxacin treatment failure in typhoid fever case, Pakistan," Emerging Infectious Diseases, vol. 9, no. 12, pp. 1621-1622, 2003.

6. GBD results tool. (2019). Accessed: March 18, 2019: http://ghdx.healthdata.org/gbd-resultstool.

7. Farjana K, Zahid HI, Bhuiya MS, Yesmine S: Pattern of antibiotic use and physician's opinion about the resistance against antibiotics used for treating respiratory tract infections (RTIs) in Bangladesh: a cross sectional survey. Jahangirnagar Univ J Biol Sci. 2016, 4:917.

8. Gupta V, Singla N, Bansal N, Kaistha N, Chander J: Trends in the antibiotic resistance patterns of enteric fever isolates - a three year report from a tertiary care centre. Malays J Med Sci. 2013, 20:71-75.
9. Klemm EJ, Shakoor S, Page AJ, et al.: Emergence of an extensively drugresistant Salmonella enterica serovar typhi clone harboring a promiscuous plasmid encoding resistance to fluoroquinolones and third-generation cephalosporins. mBio. 2018, 9:e0010518. $10.1128 / \mathrm{mBio} .00105-18$

10. Nagshetty K, Channappa ST, Gaddad SM. Antimicrobial susceptibility of Salmonella Typhi in India. J Infect Dev Ctries. 2010;4(2):70-73. Published 2010 Mar 8. doi:10.3855/jidc.109

11. Ali A, Ali HA, Shah FH, Zahid A, Aslam H, Javed B. Pattern of antimicrobial drug resistance of Salmonella Typhi and Paratyphi A in a Teaching Hospital in Islamabad. J Pak Med Assoc. 2017 Mar 1;67(3):375-9.

12. Suwantarat N, Carroll KC. Epidemiology and molecular characterization of multidrug-resistant Gram-negative bacteria in Southeast Asia. Antimicrobial Resistance \& Infection Control. 2016 Dec;5(1):1-8. 
13. Hasan R, Zafar A, Abbas Z, Mahraj V, Malik F, Zaidi A. Antibiotic resistance among Salmonella enterica serovars Typhi and Paratyphi A in Pakistan (2001-2006). J Infect Dev Ctries. 2008; 2:289-94.

14. Rahman BA, Wasfy MO, Maksoud MA, Hanna N, Dueger E, House B. Multidrug resistance and reduced susceptibility to ciprofloxacin among Salmonella enterica serovar Typhi isolates from the Middle East and Central Asia. New Microbes New Infect. 2014; 2: 88-92.
15. Qamar FN, Yousafzai MT, Khalid M, Kazi AM, Lohana H, Karim S, Khan A, Hotwani A, Qureshi S, Kabir F, Aziz F. Outbreak investigation of ceftriaxoneresistant Salmonella enterica serotype Typhi and its risk factors among the general population in Hyderabad, Pakistan: a matched case-control study. The Lancet Infectious Diseases. 2018 Dec 1;18(12):1368-76.

16. Kasper MR, Sokhal B, Blair PJ, Wierzba TF, Putnam SD. Emergence of multidrug-resistant Salmonella enterica serovar Typhi with reduced susceptibility to fluoroquinolones in Cambodia. Diagnostic microbiology and infectious disease. 2010 Feb 1;66(2):207-9.

17. Als D, Radhakrishnan A, Arora $\mathrm{P}$, Gaffey MF, Campisi S, Velummailum R, Zareef F, Bhutta ZA. Global trends in typhoidal salmonellosis: a systematic review. The American journal of tropical medicine and hygiene. 2018 Sep 6;99(3_Suppl):10-9. 